\title{
Low albumin levels are associated with mortality in the critically ill: A retrospective observational study in a multidisciplinary intensive care unit
}

\author{
A K Atrash, MB ChB, DA (SA), FCA (SA); K de Vasconcellos, MB ChB, DA (SA), FCA (SA), Cert Crit Care (SA) \\ Discipline of Anaesthesiology and Critical Care, School of Clinical Medicine, University of KwaZulu-Natal, Durban, South Africa
}

Corresponding author: A K Atrash (ashraf11_4@yahoo.com)

\begin{abstract}
Background. Albumin is a determinant of plasma colloid oncotic pressure and buffering capacity. It is a carrier protein for drugs and is important for normal functioning of the glycocalyx. Hypoalbuminaemia is common in the critically ill and has been associated with adverse outcomes. The association between hypoalbuminaemia and outcome has not been specifically explored in the South African context.

Objectives. To determine whether albumin levels on admission and changes in albumin levels were associated with intensive care unit (ICU) mortality in a heterogenous critically ill population.

Methods. This was a retrospective observational study of 247 adult patients who were admitted to a multidisciplinary ICU. Albumin levels were measured on admission and 48 hours later, alongside other biochemical and clinical parameters to determine whether they were predictive of ICU mortality.

Results. The lowest level of albumin on admission was $8 \mathrm{~g} / \mathrm{L}$ and the highest was $43 \mathrm{~g} / \mathrm{L}$. The incidence of hypoalbuminaemia (using the laboratory reference range) was $93.9 \%$ on admission and $99.4 \%$ at 48 hours. Receiver operating characteristic curve analysis provided an optimal albumin cut-off of $18.5 \mathrm{~g} / \mathrm{L}$. Using this cut-off, hypoalbuminaemia at admission and at 48 hours was associated with increased ICU mortality. Hypoalbuminaemia at admission was an independent predictor of mortality using multivariable analysis (OR 3.74; 95\% confidence interval $1.87-4.48)$.

Conclusion. Hypoalbuminaemia is associated with increased ICU mortality. There is currently no evidence to support the use of albumin replacement therapy. Further research is required to determine its role in critically ill patients.

Keywords. albumin; critical care; mortality.
\end{abstract}

South Afr J Crit Care 2020;36(2):74-79. https://doi.org/10.7196/SAJCC.2020.v36i2.422

Contribution of the study. Hypoalbuminaemia is common in critically ill South African (SA) patients and is associated with increased ICU mortality. This has not been well explored in the SA setting. We found that an optimal albumin cut-off was $18.5 \mathrm{~g} / \mathrm{L}$, which was much lower than the limits of the laboratory reference range.

Albumin is the major component of plasma proteins. ${ }^{[1,2]}$ It modulates microvascular permeability, maintains oncotic pressure, acts as a buffer, transports cations, prevents platelet aggregation and assists with binding toxins. ${ }^{[2]}$ Albumin binds to drugs and has a significant effect on the half-life and action of these drugs. ${ }^{[2]}$ In addition, serum albumin is used in assessment of the nutritional status of both acute and chronically ill patients. ${ }^{[3]}$

Total body albumin measures about $3.5-5.0 \mathrm{~g} / \mathrm{kg}$ of body weight. ${ }^{[2]}$ The plasma compartment contains $42 \%$ of the total body albumin, the rest being in the extravascular components. ${ }^{[2]}$ The liver controls the synthesis of albumin, and the rate of synthesis is controlled by nutritional intake more than other hepatic proteins. ${ }^{[2]}$ Therefore, fasting reduces albumin synthesis, but omitting protein from the diet causes a greater decrease in albumin synthesis. ${ }^{[2]}$ The degradation of albumin is about $14 \mathrm{~g} / \mathrm{day}$, or $5 \%$ of daily whole-body protein turnover in a $70 \mathrm{~kg}$ adult. ${ }^{[2]}$

The rate of synthesis and degradation and the distribution of albumin between the intravascular and extravascular compartments is altered in the critically ill. ${ }^{[2]}$ There is often a dramatic decrease in serum albumin concentration during critical illness. ${ }^{[2]}$ Serum albumin levels increase as recovery from illness progresses. ${ }^{[2]}$ The alteration of albumin distribution in critical illness is related, at least in part, to an increase in capillary leakage due to a dysfunction of the endothelial barrier. ${ }^{[2]}$ This occurs after major surgical stress and during sepsis, and results in leakage of, and loss of proteins into the interstitial space. ${ }^{[2]}$

A number of studies have attempted to describe the incidence of hypoalbuminaemia and determine if there is an association between hypoalbuminaemia and outcome. ${ }^{[4-11]}$ These studies have varied in their populations, definitions of hypoalbuminaemia and outcomes of interest. ${ }^{[4-11]}$

The frequency of hypoalbuminaemia in adult hospitalised patients (serum albumin levels $<35 \mathrm{~g} / \mathrm{L}$ ) has been reported to be $21 \%$ at the time of admission. Herrmann et al. ${ }^{[4]}$ reported an association between low serum albumin within 48 hours of admission and increased length of stay (LOS) and in-hospital mortality. Similar findings were reported in surgical populations. ${ }^{[5,6]}$ A preoperative albumin level of $<40 \mathrm{~g} / \mathrm{L}$ 
in patients undergoing radical cystectomy was associated with wound complications, increased neurological deficits and increased 90-day mortality. ${ }^{[5]}$ In patients undergoing colorectal cancer surgery, a serum albumin $<34.5 \mathrm{~g} / \mathrm{L}$ was associated with increased LOS, and in patients with gastrointestinal malignancies, a value below $32 \mathrm{~g} / \mathrm{L}$ was associated with increased mortality risk. ${ }^{[6]}$ In patients with sepsis, a serum albumin level of $<29.2 \mathrm{~g} / \mathrm{L}$ on admission was associated with increased 28-day mortality. ${ }^{[7]}$ Similarly, an association was found between serum albumin level and increased risk of intensive care unit (ICU) admission and 30-day mortality in patients with community-acquired pneumonia, and the risk appeared to increase with lower albumin levels. ${ }^{[8]}$

While the above studies may have included critically ill patients, they were not ICU-specific studies. In this regard, McCluskey et al. ${ }^{[9]}$ found that serum albumin measured 24 hours following ICU admission was a better prognostic indicator than admission serum albumin. Also, they found that serum albumin concentration measured 24 - 48 hours following ICU admission was as accurate a prognostic indicator as the admission acute physiology and chronic health evaluation (APACHE II) score. ${ }^{[9]}$ Yap et al. ${ }^{[10]}$ found that serum albumin had a low sensitivity and specificity to predict hospital mortality. They also found that a combination of serum albumin concentration with APACHE II did not improve the accuracy of outcome prediction over that of APACHE II alone. ${ }^{[10]}$ Kendall et al. ${ }^{[1]}$ found that admission albumin level, serum albumin trend and lowest serum albumin were significant predictors of mortality in ICU patients with sepsis. The probability of survival decreased by $63.4 \%$ when admission serum albumin was $\leq 24.5 \mathrm{~g} / \mathrm{L}$, and by $76.4 \%$ when the lowest serum albumin was $\leq 14.5 \mathrm{~g} / \mathrm{L}$. ${ }^{[1]}$

While the above data suggest an association between hypoalbuminaemia and adverse outcomes, questions remain about the optimal albumin cut-off point, and whether the association holds true in critically ill patients. Furthermore, none of the studies above have been performed in sub-Saharan Africa. With our combined burden of sepsis, trauma, HIV, tuberculosis and malnutrition, there are many unknowns with respect to serum albumin in the critically ill. ${ }^{[12-16]}$ It is unclear what the prevalence is of hypoalbuminaemia, what the kinetics are of albumin in our setting, and what association (if any) exists between serum albumin levels and ICU outcome. In a setting with endemic malnutrition and chronic diseases such as HIV and tuberculosis, does serum albumin have the same association with outcome as in other settings, and even if so, are our thresholds potentially different?

\section{Methods}

This is a retrospective observational study of patients admitted to a multidisciplinary, intensivist-run ICU in a tertiary academic hospital in Durban, South Africa (SA). The study consisted of an analysis of relevant data collected during a previous study, 'Hyperchloraemia as a predictor of outcome in critically ill patients' that was approved by the University of KwaZulu-Natal Biomedical Research Ethics Committee (ref. no. BE492/15). ${ }^{[16]}$

We included consecutive adult patients admitted to the ICU from 26 September 2015 to 9 May 2016. In all, data were collected for 250 adult patients. Approval to use the data for the current study was obtained from the Health Research Committee of the KwaZulu-Natal Department of Health, the study hospital and the University of KwaZulu-Natal Biomedical Research Ethics Committee (ref. no. BE279/19).

All adult ( $\geq 18$ years) patients admitted to the study ICU with admission biochemical data were eligible for inclusion in the original study. Exclusion criteria included lack of admission biochemical data (in particular admission serum albumin and chloride), end-stage renal disease (ESRD), readmission or transfer from another ICU, prior ICU admission, admission with established acute kidney injury (AKI) requiring emergent renal replacement therapy (RRT) and moribund patients. The sample size was pragmatic as previously collected data were utilised. However, to detect a difference of $20 \%$ in mortality, a sample size of 186 was required in the hypoalbuminaemic v. nonhypoalbuminaemic group $(\alpha=0.05$, power $=80 \%)$.

Hypoalbuminaemia was initially (for data collection and initial analysis) defined as a serum albumin below the laboratory reference range $(<35 \mathrm{~g} / \mathrm{L})$. Hypoalbuminaemia was also subsequently defined as a serum albumin below the optimal discriminatory point using a receiver operating characteristic (ROC) curve for the study outcome (ICU mortality). Shock was defined as the need for inotropic support or vasopressors. The Surviving Sepsis campaign guidelines of 2012 were used to define sepsis, as this was the definition at the time of the study commencement. ${ }^{[17]}$ Kidney Disease Improving Global Outcomes criteria were used to define AKI using RRT and serum creatinine criteria. ${ }^{[18]}$ Urine output criteria were not used because the patient weights were not accurately known. The lowest known creatinine prior to ICU admission was taken as the baseline, and if unknown, the modification of diet in renal disease equation was used to calculate the assumed baseline. ${ }^{[19]}$

Statistical analysis was performed using SPSS Statistics for Windows version 25.0 (IBM Corp., USA). Categorical data were analysed using descriptive statistics and presented as percentages and 95\% confidence intervals (CI), where appropriate. Categorical data were analysed using the Fisher's exact test or Pearson's $\chi^{2}$ test, where appropriate. Continuous data were analysed using descriptive statistics and presented as mean (standard deviation (SD)) when the distribution was normal, and median (interquartile range (IQR)) when there was a non-Gaussian distribution. These data were compared using independent samples ( $t$-test or Mann-Whitney U-test, respectively). All risk factors were entered into multivariable analysis using binary logistic regression. Two analyses were performed, one using demographic data and categorical biochemical data, and the other with demographic data and continuous biochemical data. The ROC curve was used to determine the optimal cut-off for serum albumin for the categorical analysis. The decision to run separate analyses for continuous and categorical data was to avoid multicollinearity and to present results that are both more practical for the clinician (categorical data) and more robust statistically (continuous data). In addition, this approach allowed for a minimum of five events per variable to reduce overfitting of the model. A backward stepwise modelling technique was used based on likelihood ratios, with entry and removal probabilities set at 0.05 and 0.1 , respectively. The odds ratio (OR) for the primary outcome and the $95 \% \mathrm{CI}$ were reported. The variance inflation factor was calculated for continuous data, and the Hosmer-Lemeshow test was performed for each analysis. A variance inflation factor of 10 was deemed the maximum acceptable value.

A ROC curve was constructed for admission serum albumin and ICU mortality. The optimal cut-off point was established using the Youden index.

\section{Results}

We originally included 250 patients in the study; however, 3 were excluded since they had no albumin data on admission. The demographic and admission biochemical data for the entire cohort, and their association with ICU mortality, are shown in Table 1. More than one-third of the patients $(n=96 / 247)$ died in the ICU, resulting in a mortality rate of $38.9 \%$. The median (IQR) LOS in the ICU was $3(2$ - 6) days. 
The ROC curve for admission serum albumin and mortality is shown in Fig. 1. The area under the curve (AUC) was 0.61 (95\% CI 0.53 - 0.68; $p=0.005$ ). The optimal cut-off point for serum albumin was $18.5 \mathrm{~g} / \mathrm{L}$ with a sensitivity of 0.52 and specificity of 0.69 .

Further details on admission, albumin values at 48 hours and the change in serum albumin over the first 48 hours of ICU admission are provided in Table 2. The lowest admission albumin was $8 \mathrm{~g} / \mathrm{L}$ and the highest was $43 \mathrm{~g} / \mathrm{L}$. The serum albumin data at 48 hours were available for 159 patients, and the lowest value was $8 \mathrm{~g} / \mathrm{L}$ while the highest was $36 \mathrm{~g} / \mathrm{L}$.

We found that the mortality rate increased while serum album levels decreased ( $p=0.037$ ), demonstrating a dose response (Fig. 2).

The multivariate analyses of categorical and continuous biochemical variables and ICU outcomes are shown in Tables 3 and 4. Serum albumin level on admission was found to be an independent predictor of ICU mortality in both categorical and continuous analyses. The variance inflation factor was $<5$ for all continuous variables. The Hosmer-
Lemeshow test results were $p=0.970$ and $p=0.835$ for the categorical and continuous models, respectively.

\section{Discussion}

The study described serum albumin levels in a critically ill SA patients and explored an association between serum albumin levels and ICU mortality. To the authors' knowledge, this is the first study of its kind in sub-Saharan Africa. The study cohort had a median age of 35 years, which is notably younger than that of previous studies from other geographic regions. ${ }^{[3,5,20]}$ The incidence of sepsis was high $(44.9 \%)$, which reflects the significance of infectious disease in our setting. The high incidence of ICU mortality (38.9\%), shock on admission (53.4\%) and AKI (49\%) reflect the severity of illness in our patients compared with other studies. ${ }^{[7,20,21,22]}$ Our study included a high proportion of nonoperative (49.4\%) and medical (38.9\%) patients that have previously been inadequately represented in the literature.

Table 1. Demographic and biochemical data for entire cohort

\begin{tabular}{|c|c|c|c|c|}
\hline & $\begin{array}{l}\text { Total, } \\
n(\%)^{\star}\end{array}$ & $\begin{array}{l}\text { Discharged alive, } \\
n(\%)^{\star}\end{array}$ & $\begin{array}{l}\text { Died in ICU, } \\
n(\%)^{\star}\end{array}$ & $p$-value \\
\hline Age, median (IQR) & $35(25-53)$ & $31(24-45)$ & $45(29-62)$ & $<0.001$ \\
\hline Male gender & $137(55.5)$ & $80(53.0)$ & $57(59.4)$ & 0.324 \\
\hline \multicolumn{5}{|l|}{ Admitting discipline } \\
\hline Medicine & $96(38.9)$ & $56(37.1)$ & $40(41.7)$ & \multirow[t]{3}{*}{0.094} \\
\hline $\mathrm{O} \& \mathrm{G}$ & $26(10.5)$ & $21(13.9)$ & $5(5.2)$ & \\
\hline Surgery & $125(50.6)$ & $74(49.0)$ & $51(53.1)$ & \\
\hline \multicolumn{5}{|l|}{ Primary diagnosis } \\
\hline Infectious & $60(24.3)$ & $30(19.9)$ & $30(31.3)$ & \multirow[t]{3}{*}{0.106} \\
\hline Non-communicable & $128(51.8)$ & $81(53.6)$ & $47(49.0)$ & \\
\hline Trauma & $59(23.9)$ & $40(26.5)$ & $19(19.8)$ & \\
\hline Sepsis on admission & $111(44.9)$ & $56(37.1)$ & $55(57.3)$ & 0.002 \\
\hline Shock on admission & $132(53.4)$ & $65(43.0)$ & $67(69.8)$ & $<0.001$ \\
\hline Postoperative & $125(50.6)$ & $82(54.3)$ & $43(44.8)$ & 0.145 \\
\hline Admission albumin (g/L), median (IQR) & $21(16.0-28.0)$ & $23(17.0-29.0)$ & $18(15.0-28.0)$ & 0.005 \\
\hline Admission hypoalbuminaemia & $231(93.9)$ & $139(92.7)$ & $92(95.8)$ & 0.311 \\
\hline Admission chloride (mmol/L), median (IQR) & $106(102.0-110.0)$ & $106(102.0-110.0)$ & $105(102.00-109.0)$ & 0.438 \\
\hline \multicolumn{5}{|l|}{ Admission chloride category } \\
\hline Normal & $125(50.6)$ & $77(51.0)$ & $48(50.0)$ & \multirow[t]{3}{*}{0.953} \\
\hline Hypochroraemia & $19(7.7)$ & $11(7.3)$ & $8(8.3)$ & \\
\hline Hyperchloraemia & $103(41.7)$ & $63(41.7)$ & $40(41.7)$ & \\
\hline Admission sodium (mmol/L), median (IQR) & $138(135.0-141.0)$ & $138(135.0-140.0)$ & $139(135.0-141.0)$ & 0.149 \\
\hline \multicolumn{5}{|l|}{ Admission sodium category } \\
\hline Normal & $155(62.8)$ & $96(63.6)$ & $59(61.5)$ & \multirow[t]{3}{*}{0.207} \\
\hline Hyponatraemia & $75(30.4)$ & $48(31.8)$ & $27(28.1)$ & \\
\hline Hypernatraemia & $17(6.9)$ & $7(4.6)$ & $10(10.4)$ & \\
\hline Admission base excess (mmol/L), median (IQR) & $-5.1(-9.30-0.50)$ & $-4.5(-8.70-1.00)$ & $-6.3(-10.70--0.10)$ & 0.072 \\
\hline \multicolumn{5}{|l|}{ Admission base excess category } \\
\hline Normal & $45(18.4)$ & $28(18.9)$ & $17(17.7)$ & \multirow[t]{3}{*}{0.347} \\
\hline Metabolic acidosis & $156(63.9)$ & $90(60.8)$ & $66(68.8)$ & \\
\hline Metabolic alkalosis & $43(17.6)$ & $30(20.3)$ & $13(13.5)$ & \\
\hline Admission pH, median (IQR) & $7.31(7.21-7.39)$ & $7.34(7.24-7.40)$ & $7.25(7.15-7.37)$ & $<0.001$ \\
\hline \multicolumn{5}{|l|}{ Admission $\mathrm{pH}$} \\
\hline Normal & $74(30.3)$ & $53(35.8)$ & $21(21.9)$ & \multirow[t]{3}{*}{0.015} \\
\hline Acidaemia & $148(60.7)$ & $79(53.4)$ & $69(71.9)$ & \\
\hline Alkalaemia & $22(9.0)$ & $16(10.8)$ & $6(6.3)$ & \\
\hline Admission lactate (mmol/L), median (IQR) & $2.6(1.40-5.50)$ & $2.2(1.10-4.50)$ & $3(1.80-6.60)$ & 0.001 \\
\hline Admission hyperlactataemia & $147(61.0)$ & $80(54.8)$ & $67(70.5)$ & 0.014 \\
\hline Admission creatinine $(\mu \mathrm{mol} / \mathrm{mL})$, median (IQR) & $106(73.0-155.0)$ & $90(66.00-128.0)$ & $122(89.0-180.0)$ & $<0.001$ \\
\hline AKI on admission & $121(49.0)$ & $58(38.4)$ & $63(65.6)$ & $<0.001$ \\
\hline
\end{tabular}


Serum hypoalbuminaemia as defined by the laboratory reference range was almost ubiquitous, occurring in all but $6.1 \%(n=15)$ of the patients on admission, and all but $0.6 \%(n=1)$ at 48 hours. There was no statistically significant association between hypoalbuminaemia and ICU mortality using the standard reference range. This is most likely a statistical phenomenon (due to the high incidence of hypoalbuminaemia), but may also reflect differences in dose response in our patients. In this

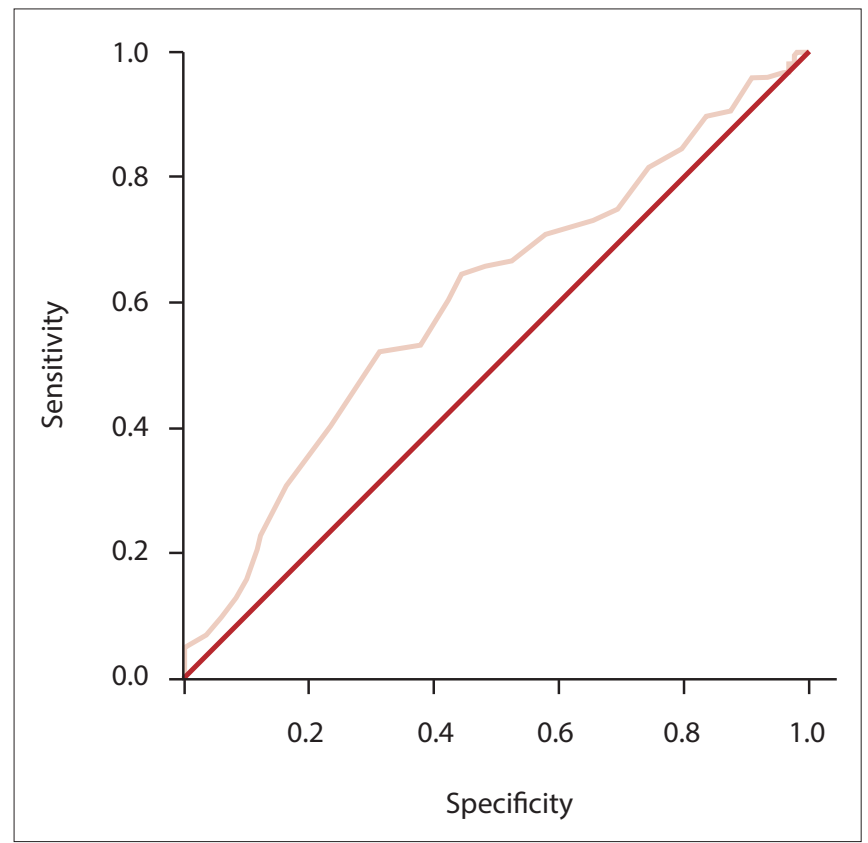

Fig. 1. Receiver operating characteristic curve of admission serum albumin and ICU mortality.

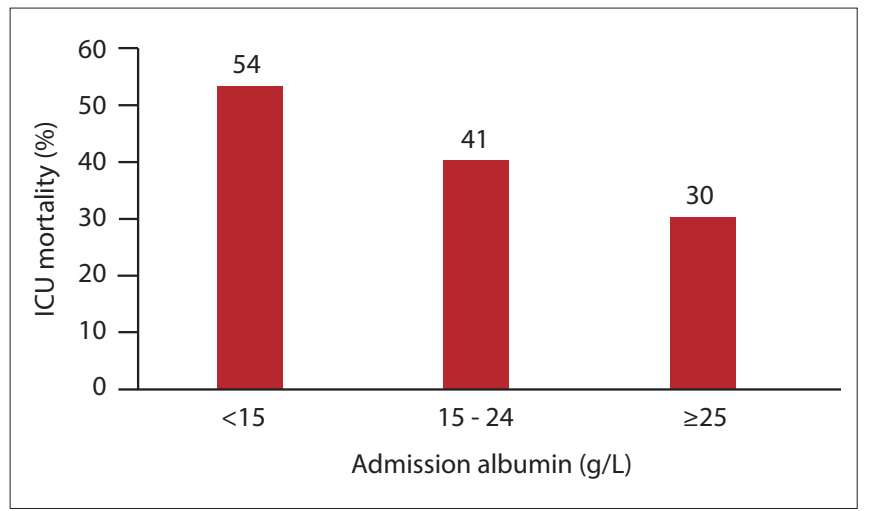

Fig. 2. ICU mortality and admission albumin category. regard, the ROC indicated an optimal cut-off point of $18.5 \mathrm{~g} / \mathrm{L}$. This is lower than the cut-offs previously reported in the literature. ${ }^{[7,23]}$ Yin et al. ${ }^{[7]}$ found that an optimal cut-off value of $29.2 \mathrm{~g} / \mathrm{L}$ and serum albumin levels $<29.2 \mathrm{~g} / \mathrm{L}$ on admission were identified as an independent risk factor for 28-day mortality in patients with sepsis. A similar finding was reported by Qian et al., ${ }^{[23]}$ who showed that a serum albumin level of $24.5 \mathrm{~g} / \mathrm{L}$ was an optimal cut-off value, and a serum albumin level $<24.5 \mathrm{~g} / \mathrm{L}$ was associated with poor survival in critically ill patients with septic shock. ${ }^{[23]}$ This may reflect potential differences in baseline severity of illness, chronic malnutrition and differences in dose response in different populations.

While the AUC and the sensitivity and specificity indicate at best a moderate ability of hypoalbuminaemia (using the cut-off point of $18.5 \mathrm{~g} / \mathrm{L}$ ) to predict ICU mortality, hypoalbuminaemia and serum albumin remain independent predictors of ICU mortality on multivariate analysis. The increasing mortality with decreasing serum albumin level also suggests a dose response curve, providing further evidence of a potentially biologically important association between serum albumin and ICU outcome. Taken together, these factors suggest that hypoalbuminaemia is of prognostic importance in critically ill patients in the SA setting. They also indicate the need to use locally relevant cut-offs when interpreting biomarkers in the clinical setting. The performance of serum albumin as an individual biomarker is not adequate to triage patients, and this highlights the complex multifaceted nature of outcome prediction in critical care. Serum albumin may, however, play a role in future outcome prediction models that may be developed in the SA setting, and further research should be conducted to explore this premise. The use of serum albumin at appropriate clinical cut-offs to direct specific therapy (e.g. albumin therapy) should also be explored.

There was no statistically significant association between albumin kinetics from admission to 48 hours and ICU mortality. This contradicts the findings of McCluskey et al. ${ }^{[9]}$ who demonstrated a prognostic value of serial serum albumin measurements in patients admitted to an ICU. This may reflect the already very low albumin levels on admission in this study cohort, delayed ICU admissions and lack of statistical power, given that only 159 patients had albumin level data at 48 hours.

While the association between low albumin levels and ICU mortality have been demonstrated in this and other studies, a key question for clinicians treating critically ill patients is whether this finding is indicative of causation or association. While albumin supplementation is used in clinical practice, there is little evidence to support this practice. The Albios trial failed to show a benefit of supplemental albumin in patients with sepsis and septic shock. ${ }^{[24]}$ Recommendations from Vincent et al. ${ }^{[25]}$

Table 2. Additional albumin variables and ICU mortality

\begin{tabular}{lllll}
\hline & Total, $\boldsymbol{n}(\%)^{*}$ & Discharged alive, $\boldsymbol{n}(\%)$ & Died in ICU, $\boldsymbol{n}(\%)$ & $\boldsymbol{p}$-value \\
\hline Admission hypoalbuminaemia using ROC cut-off $\leq 18.5$ & $97(39.3)$ & $47(31.1)$ & $50(52.1)$ & 0.001 \\
Serum albumin (48 hours), median (IQR) & $20(15.00-23.00)$ & $20(16.00-23.00)$ & $17(14.00-21.00)$ & 0.012 \\
Hypoalbuminaemia at 48 hours & $158(99.4)$ & $113(99.1)$ & $45(100.0)$ & 0.529 \\
Hypoalbuminaemia using ROC cut-off $\leq 18.5$ at 48 hours & $73(45.9)$ & $46(40.4)$ & $27(60.0)$ & 0.025 \\
Change in albumin at 48 hours, median (IQR) & $3(0.00-6.00)$ & $3(0.00-5.00)$ & $4(1.00-7.00)$ & 0.108 \\
Change in albumin at 48 hours & & & \\
$\quad$ No change & $14(8.8)$ & $12(10.5)$ & $2(4.4)$ & 0.476 \\
$\quad$ Decrease & $118(74.2)$ & $83(72.8)$ & $35(77.8)$ & $8(17.8)$ \\
$\quad$ Increase & $27(17.0)$ & $19(16.7)$ & & \\
ICU = intensive care unit; ROC = receiver operating characteristic, IQR = interquartile range. & & &
\end{tabular}


Table 3. Binary logistic regression using categorical predictor variables

\begin{tabular}{lll}
\hline Variable & OR $(\mathbf{9 5} \% \mathbf{C I})$ & $\boldsymbol{p}$-value \\
\hline Age & $1.035(1.017-1.053)$ & $<0.001$ \\
Shock on admission & $2.626(1.393-4.950)$ & 0.003 \\
Postoperative & $0.302(0.148-0.617)$ & 0.001 \\
Admission hypoalbuminaemia & $3.739(1.870-7.478)$ & $<0.001$ \\
(using cut-off $\leq 18.5)$ & & 0.051 \\
Admission acid-base status & & 0.03 \\
Admission acidaemia & $2.127(1.076-4.205)$ & 0.853 \\
Admission alkalaemia & $0.894(0.274-2.918)$ & \\
& & \\
OR = odds ratio; CI = confidence interval. &
\end{tabular}

Table 4. Binary logistic regression using continuous predictor variables

\begin{tabular}{lll}
\hline & OR $(\mathbf{9 5} \% \mathbf{C I})$ & $\boldsymbol{p}$-value \\
\hline Age & $1.030(1.012-1.048)$ & 0.001 \\
Shock on admission & $2.187(1.133-4.221)$ & 0.020 \\
Postoperative & $0.381(0.184-0.791)$ & 0.010 \\
Admission pH & $0.007(0.001-0.104)$ & $<0.001$ \\
Admission sodium & $1.108(1.022-1.201)$ & 0.013 \\
Admission chloride & $0.894(0.836-0.956)$ & 0.001 \\
Admission albumin & $0.917(0.873-0.963)$ & 0.001 \\
OR = odds ratio; CI = confidence interval. &
\end{tabular}

stated that albumin administration, although unlikely to cause harm in most patients, should be reserved for use in specific groups of patients in whom there is evidence of benefit. Currently, it is unclear which, if any, groups of patients will benefit from albumin therapy. Future studies need to focus on identifying patients who will benefit from albumin therapy, determining if there are albumin cut-offs that may identify patients who would benefit from albumin therapy, determine the dosage of albumin solution and identify appropriate albumin level targets that are needed to improve outcomes. These studies should include populations in sub-Saharan Africa and other settings where malnutrition and chronic inflammatory diseases such as tuberculosis and HIV may conceivably lead to differences in response. An important consideration that needs to be kept in mind in any discussion regarding albumin therapy in resource-limited settings is the high costs. This cost and the ethical considerations of social justice need to be considered in the design of further studies and in clinical practice.

\section{Study limitations}

The retrospective observational nature of this study has inherent limitations. A nutritional assessment was not included as part of the study, and as such, the nutritional status of the patients is unknown and the relative contribution of malnutrition v. acute inflammation to hypoalbuminaemia could not be evaluated. Furthermore, there were no data recorded regarding fluid therapy, especially intravenous albumin treatment during hospitalisation. The study ICU does not, however, advocate use of albumin, and therefore few, if any, patients would have received this therapy. The study did not exclude postpartum or pregnant patients, which is a potential confounder, as serum albumin level may be low in these patients, but the number of these patients was small and unlikely to make a difference in the findings. The patients were not stratified by severity of illness because there was no formal severity of illness scoring recorded in the patients' records. This may have been a possible confounder, but there is no reason to suspect that this would have changed the findings of the study. Patients with ESRD and those who needed emergent haemodialysis were excluded from the study to avoid confounding the biochemical tests. However, all other patients with AKI were included, which allowed this important predictor of ICU outcome to be evaluated.

\section{Conclusion}

Hypoalbuminaemia is a common finding in a heterogenous population of critically ill SA patients, and is associated with increased ICU mortality. There was an association between increasing severity of hypoalbuminaemia defined using a specific cut-off point, and ICU mortality. While administration of albumin is an attractive potential therapeutic intervention, there is no evidence to support this practice currently. Further research is required to evaluate the role of albumin as part of a comprehensive severity of illness model, and to evaluate the potential role of different albumin cut-offs in therapeutic trials.

Declaration. This study was conducted in partial fulfilment of an MMed degree. Acknowledgements. None.

Author contributions. AKA contributed to study design, data interpretation and drafting of the article. KdV contributed to study design, data analysis and review of the article. Both authors approved the article for publication.

Funding. None.

Conflicts of interest. None.

1. Limaye K, Yang JD, Hinduja A. Role of admission serum albumin levels in patients with intracerebral hemorrhage. Acta Neurologica Belgica 2016;116(1):27-30. https://doi. org/10.1007\%2Fs13760-015-0504-2

2. Nicholson J, Wolmarans M, Park GJ. The role of albumin in critical illness. Br J Anaesth 2000;85(4):599-610. https://doi.org/10.1093\%2Fbja\%2F85.4.599

3. Jin S, Bochicchio GV, Joshi M, Bochicchio KJT. Admission serum albumin is predicitve of outcome in critically ill trauma patients. Am Surg 2004;70(12):1099.

4. Herrmann FR, Safran C, Levkoff SE, Minaker KL. Serum albumin level on admission as a predictor of death, length of stay, and readmission. Arch Intern Med 1992;152(1):125-130. https:// doi.org/10.1001\%2Farchinte.152.1.125

5. Garg T, Chen LY, Kim PH, Zhao PT, Herr HW, Donat SM. Preoperative serum albumin is associated with mortality and complications after radical cystectomy. BJU Inter 2014;113(6):918923. https://doi.org/10.1111\%2Fbju. 12405

6. Gohil R, Rishi M, Tan BH. Pre-operative serum albumin and neutrophil-lymphocyte ratio are associated with prolonged hospital stay following colorectal cancer surgery. Br J Med Medic Res 2014;4(1):481-487. https://doi.org/10.9734\%2Fbimmr\%2F2014\%2F5444

7. Yin M, Si L, Qin W, et al. Predictive value of serum albumin level for the prognosis of severe sepsis without exogenous human albumin administration: A prospective cohort study. J Intens Care Med 2018;33(12):687-694. https://doi.org/10.1177/0885066616685300

8. Viasus D, Garcia-Vidal C, Simonetti A, et al. Prognostic value of serum albumin levels in hospitalised adults with community-acquired pneumonia. J Infect 2013;66(5):415-423. https:// doi.org/10.1016\%2Fj.jinf.2012.12.007

9. McCluskey A, Thomas A, Bowles B, Kishen RJA. The prognostic value of serial measurements of serum albumin concentration in patients admitted to an intensive care unit. Anaesthesia 1996;51(8):724-727. https://doi.org/10.1111\%2Fj.1365-2044.1996.tb07883.x

10. Yap F, Joynt G, Buckley T, Wong E. Association of serum albumin concentration and mortality risk in critically ill patients. Anaesth Intensive Care 2002;30(2):202-207. https://doi.org/10.1177 $\% 2 \mathrm{~F} 0310057 \times 0203000213$

11. Kendall H, Abreu E, Cheng A-L. Serum albumin trend is a predictor of mortality in ICU patients with sepsis. Bio Res Nurs 2019;21(3):237-244. https://doi.org/10.1177\%2F1099800419827600

12. Wise R, de Vasconcellos K, Skinner D, et al. Outcomes 30 days after ICU admission: The 30 DOS study. S Afr J Anaesth Analg 2017;23(6):139-144. https://doi.org/10.1080\% 2F22201181.2017.1402553

13. Skinner DL, De Vasconcellos K, Wise R, et al. Critical care admission of South African (SA) surgical patients: Results of the SA surgical outcomes study. S Afr Med J 2017;107(5):411-419. https://doi.org/10.7196\%2Fsami.2017.v107i5.11455

14. Naidoo K, De Vasconcellos K, Skinner D. Procalcitonin kinetics in the first 48 hours of ICU admission is associated with higher mortality in critically ill patients with community-acquired pneumonia in a setting of high HIV prevalence. S Afr J Anaesth Analg 2018;24(5):128-134. https://doi.org/10.1080\%2F22201181.2018.1514787

15. Gopalan P, De Vasconcellos KJ. Factors influencing decisions to admit or refuse patients entry to a South African tertiary intensive care unit. S Afr Med J 2019;109(9):645-651. https://doi. org/10.7196\%2Fsamj.2019.v109i9.13678

16. De Vasconcellos K, Skinner DL. Hyperchloraemia is associated with acute kidney injury and mortality in the critically ill: A retrospective observational study in a multidisciplinary intensive care unit. J Critical Care 2018;45:45-51. https://doi.org/10.1016\%2Fj.jcrc.2018.01.019

17. Dellinger RP, Levy MM, Rhodes A, et al. Surviving sepsis campaign: International guidelines for management of severe sepsis and septic shock, 2012. Intensive Care Med 2013;39(2):165-228 https://doi.org/10.1007\%2Fs00134-012-2769-8 
18. Kellum JA, Lameire N, Aspelin P, et al. Kidney disease: Improving global outcomes (KDIGO) acute kidney injury work group. KDIGO clinical practice guideline for acute kidney injury. 2012;2(1):1-138. https://doi.org/10.1038/kisup.2012.1

19. Bellomo R, Ronco C, Kellum JA, Mehta RL, Palevsky P. Acute renal failure - definition, outcome measures, animal models, fluid therapy and information technology needs: The Second International Consensus Conference of the Acute Dialysis Quality Initiative (ADQI) Group. 2004;8(4):R204. https://doi.org/10.1186/cc2872

20. Akirov A, Masri-Iraqi H, Atamna A, Shimon I. Low albumin levels are associated with mortality risk in hospitalised patients. Am J Med 2017;130(12):1465. el1-e19. https://doi.org/10.1016\%2Fj. amimed.2017.07.020

21. Kim YS, Sol IS, Kim MJ, et al. Serum albumin as a biomarker of poor prognosis in the paediatric patients in an intensive care unit. Korean J Crit Care Med 2017;32(4):347-355. https://doi. org/10.4266\%2Fkjccm.2017.00437
22. Menon V, Greene T, Wang X, et al. C-reactive protein and albumin as predictors of all-cause and cardiovascular mortality in chronic kidney disease. Kidney Inter 2005:68(2):766-772. https://doi. org/10.1111\%2Fj.1523-1755.2005.00455.x

23. Qian S-Z, Jin D, Chen Z-B, et al. Hypoalbuminemia, a novel prognostic factor for prediction of longterm outcomes in critically ill patients with septic shock. Int J Clin Exp Med 2019;12(6):7401-7409.

24. Caironi P, Tognoni G, Masson S, et al. Albumin replacement in patients with severe sepsis or septic shock. New Engl J Med 2014;370(15):1412-1421. https://doi.org/10.1056\%2Fnejmoal305727

25. Vincent J-L, Russell JA, Jacob M, et al. Albumin administration in the acutely ill: What is new and where next? Critical Care 2014;18(4):231. https://doi.org/10.1186\%2Fcc13991

Accepted 1 April 2020 